\title{
Orientación para el desarrollo de la carrera: Instrumento para la exploración
}

Counseling for carrer development: exploration instrument

\author{
Volumen 22 Número 1 \\ Enero - Abril \\ pp. 1-29
}

Osvaldo Murillo Aguilar

Citar este documento según modelo APA

Murillo Aguilar, Osvaldo. (2022). Orientación para el desarrollo de la carrera: Instrumento para la exploración. Actualidades Investigativas en Educación, 22(1), 1-29. Doi. https://doi.org/10.15517/aie.v22i1.49071 


\title{
Orientación para el desarrollo de la carrera: Instrumento para la exploración

\author{
Counseling for carrer development: exploration instrument
}

\section{Osvaldo Murillo Aguilar ${ }^{1}$}

\begin{abstract}
Resumen: La teoría del desarrollo de la carrera se enfatiza en la comprensión del proceso que realiza la persona para construir su identidad personal y profesional a lo largo de la vida. Sin embargo, en el ejercicio profesional de la Orientación, practicar dicha exploración requiere ahondar en sus experiencias, interacciones y actividades efectuadas, así como en los sentidos otorgados. Según lo anterior, este artículo tiene como finalidad operacionalizar un instrumento de exploración de corte cualitativo, donde se represente el desarrollo de la carrera de las personas, por medio de un apoyo didáctico y visual a través de un gráfico. De este modo, la intención del instrumento será favorecer el diálogo en el proceso de intervención, así como la exploración de elementos tales como: la percepción de las experiencias significativas; los recursos personales y los apoyos con que se cuenta, es decir, contribuirá a plantear la estrategia y a seguir en el proceso de intervención. Cabe resaltar que el instrumento fue desarrollado en Costa Rica y sometido a una validación cualitativa en el año 2021, con ayuda de un panel de personas expertas en Orientación, centradas en determinar sugerencias de mejora; utilidad del instrumento; viabilidad y posibles criterios para la interpretación. Como resultado, el panel experto señaló la viabilidad de la aplicación del instrumento, la facilidad de comprensión que -a partir de una perspectiva holística e integral- arroja información, para construir el perfil de la persona orientada y de esta forma fundamentar el resto del proceso de intervención. En definitiva, se concluye que la aplicación de dicho instrumento de exploración facilitará la acción orientadora, además de ser un aporte significativo en el ejercicio profesional de la Orientación y una herramienta que surge desde la visión de la disciplina en el contexto particular costarricense.
\end{abstract}

Palabras clave: desarrollo de carrera, instrumento exploratorio, orientación.

Abstract: The theory of career development emphasizes the understanding of the process carried out by the person to build their personal and professional identity throughout life. However, in the professional practice of Counseling, practicing such exploration requires delving into their experiences, interactions and activities carried out, as well as the meanings given to them. According to the above, this article aims to operationalize a qualitative exploration instrument, where the career development of people is represented, through didactic and visual support through a graph. In this way, the intention of the instrument will be to favor dialogue in the intervention process, as well as the exploration of elements such as: the perception of significant experiences; the personal resources and support available to the person, that is to say, it will help to propose the strategy to be followed in the intervention process. It should be noted that the instrument was developed in Costa Rica and subjected to qualitative validation in 2021, with the help of a panel of experts in Counseling, focused on determining suggestions for improvement; utility of the instrument; feasibility and possible criteria for interpretation. As a result, the expert panel pointed out the feasibility of applying the instrument, the ease of understanding that -from a holistic and comprehensive perspective-provides information to build the profile of the targeted person and thus base the rest of the process of intervention. In short, it is concluded that the application of said exploration instrument will facilitate the guiding action, in addition to being a significant contribution in the professional practice of Counseling and a tool that arises from the vision of the discipline in the particular Costa Rican context.

Key Words: career development, instrument, counseling,

1 Docente de la Universidad de Costa Rica, en la Escuela de Orientación y Educación
Especial, San José, Costa Rica. Orientador, Oficina de Orientación, Universidad de Costa
Rica. Maestría Académica en Ciencias de la Educación con énfasis en Orientación en el
área laboral. ORCID: https://orcid.org/0000-0002-5425-9419

Dirección electrónica: OSVALDO.MURILLO@ucr.ac.cr

Artículo recibido: 20 de agosto, 2021

Enviado a corrección: 18 de octubre, 2021

Aprobado: 22 de noviembre, 2021 


\section{Introducción}

Los recientes aportes en la teoría del desarrollo de la carrera destacan la necesidad de comprender la complejidad del comportamiento vocacional de la persona, de los significados que esta le otorga a los eventos y experiencias vividas a lo largo del ciclo vital, más que a la prescripción de carreras u ocupaciones.

En este sentido, McMahon (2014) señala la necesidad de retomar en los procesos de orientación, los significados que las personas han construido a lo largo de su vida, los cuales dan sentido a su carrera, entendida esta como todo aquello que la persona ha realizado desdelos diferentes roles asumidos durante su desarrollo. Con base en esta postura, la autora apunta que las tendencias teóricas han retomado los aportes del constructivismo y el constructivismo social.

Parafraseando a Sánchez (2017), en la actualidad se hace necesario, por la misma complejidad de los cambios sociales, económicos y laborales, que la intervención en Orientación sea capaz de analizar, desde una perspectiva holística, la totalidad de los aspectos vinculados a la carrera y cómo estos interactúan en el proceso de toma de decisiones vocacionales.

De ahí que, estas posturas, hagan hincapié en que los procesos de orientación retomen como punto medular de la intervención, la interpretación y el análisis de la historia de la persona por medio de métodos narrativos, con el fin de identificar y clarificar los significados atribuidos a los eventos más trascendentales de su vida.

No obstante, en el ejercicio de la práctica profesional se origina la duda respecto a cómo llevar a cabo estas propuestas ya que, en ocasiones, el tiempo es una variable limitante para ahondar en la narrativa de la historia de vida o también a la persona orientada se le dificulta relatar los eventos con total apertura. Debido a esto, surge la interrogante motivadora de este artículo: ¿Cómo poder analizar los eventos más significativos del desarrollo de la carrera de una persona y el sentido atribuido a ellos de forma clara y expedita?

Al respecto, considerando que los aportes teóricos antes mencionados se fundamentan cada vez más en criterios del constructivismo y constructivismo social, así como la manera en que las personas otorgan sentido a las experiencias de vida en relación con el desarrollo de la carrera, se plantea el objetivo de elaborar un instrumento facilitador para dicha indagación, el cual, más allá de presentar una escala o una representación cuantitativa de las características de la persona orientada, sea un instrumento de corte cualitativo para el diálogo en la relación 
entre la persona profesional y la orientada, donde además se aprecie como eje, tanto la trayectoria como los eventos más significativos.

De esta forma, se trata de una representación gráfica de este eje con la intención de favorecer el análisis de las vivencias de forma visual, para que tanto la persona orientadora como la orientada, adquieran de manera concisa y concreta una representación del desarrollo de carrera por medio de este apoyo didáctico para el proceso.

Para la elaboración de dicho instrumento, se partió de las contribuciones teóricas de dos enfoques complementarios, pues ambos convergen en reconocer que, para analizar el desarrollo de la carrera, resulta importante internarse en las transiciones de vida de las personas. Primero, el de autoconstrucción de la carrera, propuesto recientemente a inicios del siglo XXI por Savickas y Guichard; segundo, el enfoque de transiciones, formulado en la década de 1980 por Anderson, Goodman y Schlossberg y actualizado recientemente en 2012 por parte de las autoras.

Con el fin de profundizar en la propuesta y mostrar cómo se elaboró el instrumento, a continuación se presentan los presupuestos teóricos del estudio.

\section{Referentes teóricos}

\subsection{Comprensión del desarrollo de la carrera: Aportes del enfoque de autoconstrucción y el enfoque de transiciones}

El concepto de desarrollo de la carrera se puede ubicar en sus inicios en los estudios y aportes teóricos de Super (1969), quien la definió como la integración de los diversos roles que la persona ha asumido a lo largo de su vida, los cuáles, no necesariamente se relacionan con el aspecto académico o profesional sino también con aquellos papeles que la persona asume como madre, padre, hermano, hermana, compañero, ciudadana, entre otros.

Por otra parte, Sánchez (2017), señala que el término de carrera permite una comprensión integral de la persona, pues se refiere tanto a la vida personal como a la faceta laboral, además subraya al desarrollo de la carrera como un término más oportuno cuando hace referencia al proceso vocacional, ya que permite su comprensión; inseparable de la maduración de la identidad $\mathrm{y}$, a la vez, facilita entender la autorrealización en todas sus dimensiones. 
Lo anterior es apoyado por Bisquerra (2016, p. 41) quien señala que el concepto de desarrollo de la carrera "supone el pasar a una concepción holística de la orientación que incluya todos los aspectos de la vida".

De esta manera, se puede mencionar a esta perspectiva altamente compatible con la disciplina de la Orientación, pues comprende a la persona como un ser activo y dinámico en diferentes ámbitos y en las diversas etapas de su vida. Además, en cada experiencia que vive, influye significativamente en la construcción de su identidad, tanto personal como profesional, ya que ambas se conforman como un solo sistema.

Asimismo, esto conlleva a considerar el desarrollo de la carrera como un proceso siempre en construcción. Al respecto, Ribeiro (2013) destaca que esta característica, propicia que la carrera se encuentre a expensas de variables contextuales y personales que interactúan constantemente entre sí, como por ejemplo las habilidades personales, intereses, motivaciones, influencias familiares, sistemas económicos, instituciones educativas, organizaciones religiosas, entre otras.

Por lo anterior, se podría afirmar que el desarrollo de la carrera implica un proceso complejo y dinámico, el cual, requiere ser estudiado con aportes teóricos con las mismas cualidades, en especial, en un contexto como el actual, en donde la influencia -de los factores contextuales- se encuentra en constante cambio y evolución, dejando de lado las perspectivas prescriptivas o explicaciones lineales de la carrera.

De ahí que se requiere un enfoque facilitador para analizar la dinámica que enlaza las diversas facetas de la vida de las personas, de manera holística, característica que posee según Savickas, et al, (2009) el enfoque de la autoconstrucción de la carrera.

Ahora bien, una de las principales características de este enfoque, según lo exponen estos autores, radica en alientar a las personas a considerar simultáneamente todos los papeles destacados de su vida. Para lo cual, será necesario explorar los diferentes contextos, incluidas las experiencias del pasado y sus expectativas a futuro.

Es decir, se hace hincapié en retomar todas las facetas de las personas para comprender sus elecciones ocupacionales o profesionales e incluso su desempeño en el ámbito laboral. El enfoque teórico de la autoconstrucción de la carrera señala, desde su perspectiva sociológica, la importancia de que la relación de ayuda aborde las diferentes facetas en la vida de las personas y con ello el modo de relacionarse consigo misma y con su contexto (Guichard, 2007). 
Para este autor, a partir de las relaciones intra e interpersonales, la persona va configurando cognitivamente formas de identidad subjetiva, las cuales, se definen como maneras de verse a sí misma y a las otras personas en determinados contextos. Así, estas formas de identidad subjetivas son las que podrían determinar de alguna manera, cómo se asumen las diversas situaciones de la vida laboral e incluso la forma de cómo la persona se asume en esas circunstancias particulares.

Sin embargo, la exploración de estos aspectos podría llegar a parecer algo abstracto para ser abordado en los procesos de Orientación. Por eso, la propuesta tanto de Guichard (2007) como de Savickas (2015), propone que sea a través de la narración donde la persona pueda comentar su historia de vida por medio de diversas técnicas de expresión narrativa, las cuales, permitan identificar los intereses, motivos, experiencias, habilidades, redes de apoyo y contextos donde el individuo ha vivido, además, de un panorama lo más detallado posible de la percepción de sí misma y de las proyecciones que pueda realizar a futuro.

Para Murray citado por Guichard (2002), esta exploración es fundamental, ya que el desarrollo de la carrera se determina por las diversas experiencias que enfrenta la persona, en otras palabras, por las transiciones experimentadas en su vida y lo que estas han significado para ella. Pare este autor, las transiciones se entienden como todos aquellos acontecimientos que propician cambios en áreas como las relaciones, ocupaciones cotidianas, creencias y funciones.

No obstante, el mayor aporte en cuanto a este aspecto es la perspectiva teórica de Anderson et al., (2012), quienes afirman que una transición puede presentarse por un evento o incluso la ausencia de ciertos eventos esperados, los cuales, desencadenan una serie de cambios en los roles, actividades y relaciones interpersonales establecidas por las personas, por lo tanto, el enfoque estudia dichas situaciones y la manera en que integran estos cambios en sus vidas. Desde esta perspectiva es clave analizar la fuente donde se origina la transición (es decir el tipo de evento), el tiempo cuando ocurre el evento (gradual o sorpresivo), el afecto o manera como se valora la situación (es decir la manera en cómo lo percibe la persona) y la duración de este (permanente, temporal o incierto).

Ahora bien, desde este enfoque, una de las premisas fundamentales yace en que cada persona es responsable de dar a las situaciones o eventos un carácter de transición. Así, si una situación en particular -en la vida de una persona- no le implica generar cambios, 
probablemente esta no lo considerará una transición, por lo tanto, no la asumirá como algo significativo.

Por tanto, con lo expuesto hasta ahora sobre el enfoque de transiciones, se observan cuatro variables expuestas por Anderson et al. (2012), en su propuesta. La primera de ellas se trata del evento en sí mismo, el cual puede consistir en: una situación particular que sucediera de improviso; un evento esperado que no sucedió o un evento que aún no ha sucedido y se ha atrasado en su llegada. Estos acontecimientos se clasifican de la siguiente manera:

- Evento esperado: se trata de aquel evento anhelado y, para el cual, la persona realiza un esfuerzo para que este se lleve a cabo. Tal es el caso de una graduación o un matrimonio.

- Evento de onda: aquel evento que sucede a un tercer sujeto o, en un contexto donde la persona no tiene injerencia directa, pero el impacto del hecho es significativo para su vida porque provoca cambios en sus roles, interacciones o actividades. Por ejemplo, cuando una empresa transnacional decide cerrar algunas de sus sucursales y la persona sea despedida.

- Evento inesperado: aquel evento que impacta significativamente a una persona pero sin ser planificado o previsto. Esto puede ilustrarse como cuando alguien sufre un accidente de tránsito u ocupacional.

- No evento: aquel evento que se proyecta y se anhela, pero no llega a suceder o concretarse. Por ejemplo, no ingresar a la universidad elegida o no graduarse.

La segunda variable por considerar reside en la persona en sí misma, ya que es quien determina el impacto y la valoración concebida del evento. En este caso se trata de analizarlos recursos internos con los cuales la persona enfrenta la transición como, por ejemplo, sus habilidades, motivaciones u otros.

La tercera variable presente en una transición se refiere a las redes de apoyo donde esta persona puede recurrir ante un evento, o sea aquellos aspectos externos o del contexto con los cuales cuenta, por ejemplo, sus amistades, la familia, docentes, la institución educativa, grupos de pares, entre otros.

Por último, de acuerdo con Anderson et al. (2012), la cuarta variable es la estrategia que la persona pone en marcha para incorporar este proceso de transición a su vida, la cual puede desembocar en al menos dos consecuencias: una aceptación de los eventos sucedidos o una 
renovación de su vida a partir de los hechos; en caso contrario de no lograrse lo anterior, es posible que la persona comience a experimentar un deterioro en algunas facetas de su vida, donde la transición pase más bien a experimentarse como una grave crisis.

En resumen, el desarrollo de la carrera se puede comprender desde Orientación como un proceso de construcción continuo en el que convergen todas aquellas experiencias significativas para la persona orientada, con lugar en un contexto concreto y en interacción con las características particulares de cada uno. Para facilitar dicha comprensión, el presente artículo sugiere integrar las premisas antes descritas de la teoría de la autoconstrucción de carrera con los criterios expuestos del enfoque de transiciones, pues ambas teorías convergen en que la carrera está constituida con las transiciones experimentadas por la persona, las cuales, seguirá experimentado en su ciclo vital.

Realizar dicha integración en la práctica orientadora implica, entre otros aspectos, detenerse en la exploración de tales transiciones experimentas por la persona en su vida. Sin embargo, no es sencillo narrar su historia de vida, lo cual demanda de parte de la persona profesional en Orientación desarrollar estrategias facilitadoras de este proceso de exploración, sin demeritar la profundización requerida. Por dichas razones, a continuación se presenta un instrumento de carácter cualitativo para facilitar la operacionalización de los criterios teóricos esbozados anteriormente, los cuales, corresponden a los enfoques de autoconstrucción de carrera y transiciones, de manera tal que, la persona profesional en Orientación, cuente con un insumo práctico para su labor profesional y, a su vez, le permita ahondar en la manera cómo su orientada u orientado han construido su carrera, para así, estructurar el proceso de intervención con mayor claridad.

\subsection{Gráfico de eventos significativos en el desarrollo de la carrera: Instrumento de exploración}

El "Gráfico de eventos significativos en el desarrollo de la carrera", consiste en un instrumento de exploración de tipo cualitativo, el cual busca facilitar la identificación de los acontecimientos claves e influyentes en el desarrollo de carrera de la persona orientada, enfatiza en el significado que esta le otorga y clarifica el perfil de dicha persona. De esta forma, se pueden establecer los lineamientos del proceso de intervención, favorecer el autoconocimiento y la toma de decisiones. 
El instrumento está estructurado de manera que permite tanto a la persona profesional en Orientación como a la orientada, contar con un panorama del desarrollo de carrera, de forma que sigue la propuesta teórica de los enfoques antes expuestos centrados en la narrativa. Asimismo, para lograrlo, se toman como base los cuatro aspectos o dimensiones claves para comprender la forma en la cual las personas abordan los eventos de su vida y que, a su vez, han propiciado una transición. Tales aspectos son: el evento, los recursos personales, los apoyos y las estrategias empleadas (Anderson et al., 2012).

Si bien es cierto, la propuesta de estas autoras reside en abordar eventos o situaciones del aquí y del ahora, su aporte facilita el análisis retrospectivo de eventos, de manera que puedan traerse al presente y valorarlos en función de su influencia en el desarrollo de la carrera, lo cual, a su vez, se relaciona con el enfoque de autoconstrucción de carrera que enfatiza en el sentido que se otorga a las experiencias cómo estas ayudan a confirmar la identidad profesional u ocupacional.

El "Gráfico de eventos significativos en el desarrollo de la carrera", está organizado en dos partes, la primera, se centra en los eventos significativos, la clasificación de estos y el periodo de tiempo en que sucedieron y la segunda explora tres aspectos: los aprendizajes obtenidos por la persona de las experiencias vividas, los apoyos con los que contó para afrontar la transición y las destrezas desarrolladas al transitar por esos eventos. La siguiente tabla describe a manera de síntesis el instrumento: 
Tabla 1

Información general del Instrumento Gráfico de eventos significativos del desarrollo de carrera Secciones del Descripción

instrumento

Descripción del $\quad$ En esta primera sección se encuentra una explicación general de lo que se instrumento pretende obtener con el instrumento.

I Parte

Consiste en unas indicaciones generales para elaborar el Gráfico de Eventos Significativos.

La elaboración del Gráfico para representar: los eventos significativos, el tipo de eventos y la edad en la que estos ocurrieron.

Reflexión guiada con la persona profesional en Orientación (dos preguntas abiertas para ello)

II Parte $\quad$ La elaboración de la matriz para lo cual se exploran:

Aprendizajes que se derivan de los eventos.

Tipos de apoyos y su aporte en las transiciones.

Identificación de destrezas personales gracias a las experiencias descritas. (esto responde a una resignificación de los eventos)

Cierre $\quad$ Síntesis de la información compartida en a partir de determinar cualidades personales que se desprenden de su desarrollo de carrera.

Opinión general de la persona orientada acerca de la información que observa de sí misma.

Fuente: elaboración propia.

La finalidad de la primera parte del instrumento consiste en indagar acerca de los eventos significativos en el desarrollo de la carrera y representarlos en una imagen para facilitar su análisis. Por ejemplo, como se logra observar en el anexo 1, el eje vertical del gráfico permite ubicar el tipo de evento según lo propuesto por Anderson et al., (2012) en: esperado, inesperado, de onda o sin evento; mientras que el eje horizontal facilita su ubicación en un rango de edad concreto.

Durante esta primera etapa del ejercicio se le solicita a la persona orientada reflexionar acerca de eventos significativos en su vida hayan sido satisfactorios o no. De estos eventos se le indica seleccionar los cinco más significativos; tales acontecimientos pueden ser académicos (graduaciones, éxito en un examen, reprobar un año), profesionales (conseguir o perder un empleo, lograr o no un ascenso, obtener reconocimiento, entre otros) o personales (la separación de los padres, un paseo, una pérdida, conocer una persona, aprender a tocar un instrumento, u otros).

Posteriormente, la persona debe ubicar los cinco eventos en el Gráfico, concretamente sobre la línea horizontal para indicar la edad en la cual sucedió cada uno, pues, según lo expuesto por Healy, Mitchell y Mourton, citados por Chía de la Rosa (2018), la edad es un criterio que puede incidir en la madurez vocacional de la persona y la adaptación a los eventos durante la construcción de carrera. Una vez ubicados, se clasifica cada uno según su tipo de 
acuerdo con lo indicado en el eje vertical; para guiar a la persona, se conceptualiza brevemente cada evento según el enfoque de Transición, de manera que tanto el tipo de evento como la edad se cruzan en un punto del gráfico.

El objetivo de ubicar gráficamente -cada evento- es facilitar la reflexión acerca de los aspectos relativos a aquellos tipos de hechos más frecuentes en el desarrollo de la carrera. Por ejemplo, un gráfico donde se presenta mayoritariamente una línea horizontal, puede indicar el predominio de un tipo de evento sobre los demás o gráficos con líneas muy curvas o pronunciadas, indicarían la flexibilidad de las personas orientadas para afrontar diversos eventos en sus vidas, así como su capacidad para transitar por ellos.

De esta forma, la ayuda visual se considera un apoyo para las personas orientadas y profesionales para generar una discusión profunda sobre la historia su vida y, por ende, el desarrollo de carrera, donde ella se visualice como sujeto activo de su propio proceso, independientemente de la edad de cuando sucedieron los eventos o, incluso, de las herramientas con las que contaba en ese momento.

Asimismo, ubicar los eventos en el periodo de edad en que estos ocurrieron, permite observar la consecución o postergación de tareas del desarrollo, así como el hecho de visualizar los momentos de su vida donde tuvo mayor exposición a un tipo de evento en particular. Además, contribuye a definir cuándo se desarrollaron algunas destrezas o intereses, los cuales, favorecieron con el desarrollo de la identidad profesional u ocupacional.

La segunda parte del instrumento se presenta como una matriz de cuatro columnas. Esta, se centra en analizar, a partir de los eventos señalados, cómo se pueden desprender aprendizajes claves para el desarrollo de carrera.

La intención es propiciar que la persona orientada reflexione acerca de los principales aprendizajes adquiridos en cada uno de los eventos señalados, y la razón por la que se consideran situaciones significativas en su historia de vida.

Seguidamente, al lado de estos aprendizajes de vida, se conduce a la persona a identificar en cada evento descrito, los apoyos o recursos externos con los que contó en ese momento para llevar a cabo la transición. Entre los aspectos que se pueden señalar se encuentran la familia, las amistades, grupos, instituciones y pareja. La intención en este punto es clarificar qué aportaron y si hoy en día se pueden seguir considerando una red de apoyo para decisiones por emprender. 
Por último, una vez distinguidos los anteriores aspectos, el instrumento propicia la identificación de destrezas personales desprendidas de los aprendizajes señalados y de los aportes de sus apoyos en estos eventos. Esto se realiza con el fin de establecer un perfil de la persona orientada y utilizarlo en la organización del proceso de intervención. Al finalizar la realización del instrumento se solicita a la persona orientada reflexionar acerca de la información detallada, las emociones y pensamientos que esta le genera y lo que concluye acerca de sí misma como persona, su identidad y el desarrollo de la carrera.

Como se logra apreciar de la descripción anterior para utilizar el instrumento se requiere a la persona profesional en Orientación presente durante su aplicación, ya que, la persona orientada puede encontrarse frente a eventos que despierten alguna susceptibilidad en ella, por lo tanto, es importante la disposición del profesional para contenerle. Otro aspecto por considerar, acerca del Grafico de Eventos Significativos, en cuanto al tipo de información que se busca, se recomienda utilizarlo en población adolescente y adulta, pues en estas edades es más factible determinar eventos en el desarrollo de carrera que en poblaciones menores o de infantes.

\section{Metodología}

\subsection{Enfoque}

El enfoque seleccionado para someter el instrumento a un proceso de evaluación de contenido fue el cualitativo, realizado con personas expertas, con el fin de ser consecuente con las características del instrumento, por ser también cualitativo. Los procesos de este tipo de enfoque se caracterizan, según lo expuesto por Sánchez (2019) por centrarse en las experiencias de las personas, la semántica, los significados conceptuales y en comprender los hechos desde la perspectiva de las personas participantes. En el caso de la validación de este instrumento, se buscaba obtener una opinión experta en relación con aspectos tanto de utilidad como de viabilidad, para así ser aplicado en la población con la cual laboran cotidianamente. La metodología utilizada para la realización de este trabajo es la fenomenología, pues, de acuerdo con Fuster (2019), facilita analizar el objeto de estudio desde la experiencia de la persona participante y los significados que ella le otorgue desde su subjetividad, siendo estos la esencia de la interpretación y la descripción de los resultados.

El tipo de estudio presentado corresponde al de una validación. Donde se destaca que esta consiste, según lo expuesto por Kvale (2011), en un proceso centrado en sopesar 
argumentos a favor de la credibilidad de afirmaciones o conocimientos. Dicho proceso depende en gran medida de la calidad del conocimiento o experticia de las personas consultadas.

Dicha validación buscó obtener las impresiones o percepciones de personas expertas profesionales en Orientación (cuya descripción se realiza en el apartado 3.3) respecto al "Gráfico de eventos significativos en el desarrollo de la carrera" como instrumento de exploración, con el fin de conocer si, de acuerdo con su experiencia profesional, este es útil para su labor orientadora y si considera viable su aplicación.

\subsection{Técnica de recolección}

Para conocer dichas impresiones se recurrió a la estrategia llamada validación de grupo de expertos, según lo mencionan Robles y Rojas (2015), es una estrategia que posibilita ahondar en amplios criterios acerca de un contenido u objeto de estudio, así como la calidad de lo que se somete a juicio. Para estas autoras, la correcta aplicación de esta estrategia depende de los criterios de selección y del número adecuado de las personas participantes.

El grupo o panel de expertos forma parte de un conjunto de técnicas denominadas de consenso, las cuales según López et al. (2014), pretenden obtener un acuerdo entre especialistas acerca de un problema o tema en concreto, de manera que emitan un criterio de carácter colectivo y, por ende, de mayor validez, por lo tanto, la discusión grupal es una de sus características principales.

Por su parte, Lewthwaite y Nind (2016), enfatizan en que esta estrategia es clave para determinar las impresiones y pensamientos de las personas expertas, por medio de la narrativa surgida de su experticia, acerca de un tema atinente a su área disciplinar. De ahí que, para este estudio, centrado en la validación de un instrumento para el ejercicio de la acción orientadora, se determinó al grupo de expertos como el método para establecer dicha viabilidad, utilidad y practicidad del instrumento. De esta forma, se les diseñó una guía de preguntas generadoras, relacionadas con los criterios de viabilidad, utilidad y practicidad, para que con ella se pudiera facilitar la discusión en el grupo y, así, brindar sugerencias para mejorar su diseño. 


\subsection{Población}

De acuerdo con lo postulado por Robles y Rojas (2015) la selección de las personas para una estrategia de recolección de información como lo es el panel de expertos, en ocasiones no implica el uso de algún filtro de selección, en otras se utilizan una serie de criterios estructurados definidos previamente y también, como en este caso, es posible utilizar criterios de afinidad o cercanía entre las personas expertas e investigadoras.

Por su parte López et al. (2014), plantean como otro aspecto por considerar que el grupo de especialistas debe estar conformado por personas ajenas a la investigación. De ahí que, las personas expertas para este estudio, fueron contactadas por medio de un criterio de oportunidad, pues se encontraban inscritas en un curso de actualización profesional en Orientación Laboral. Además como grupo cumplían con cualidades idóneas para la validación del instrumento. Entre las características de las personas profesionales se pueden citar:

- Cuentan con más de dos años de experiencia en el campo profesional de la Orientación.

- Laboran en distintos contextos de intervención en Orientación tales como: educación formal privada y pública, instituto de educación técnica no formal, sistema penitenciario y organizaciones no gubernamentales.

- Laboran con población adolescente y adulta.

Por otro lado, las personas expertas, contaban con conexión a internet para la realización de la consulta, lo cual, debido a las restricciones sanitarias generadas por la pandemia de COVID19, fue indispensable para su participación. A las personas expertas se les consultó acerca de su disponibilidad para participar en el estudio y conocer sus impresiones respecto al "Instrumento de exploración cualitativa": "Gráfico de eventos significativos en el desarrollo de la carrera", donde mostraron total anuencia. Igualmente, para confirmar la participación, se les hizo llegar el formulario de "consentimiento informado", el cual firmaron todas las personas expertas. En este, se garantizaba que la información suministrada de su parte sería utilizada únicamente para los fines del presente estudio y que el tratamiento de la información se realizaría de manera confidencial.

Respecto a la cantidad de participantes, según lo exponen Robles y Rojas (2015), no hay un acuerdo unánime para su determinación, por lo tanto, la cantidad de personas expertas queda sujeta a criterios como la facilidad para acceder a ellas o la cantidad de estas conocidas en el área de estudio. De este modo, para conformar el grupo se invitó a las once personas 
profesionales en Orientación y se contó con la totalidad en el panel. Posterior a esto, se concertó un día y hora en específico para la consulta, realizada, el 5 de julio del año 2021.

La consulta consistió en dos momentos concretos: una primera etapa donde se explicaba el instrumento de exploración, su fundamentación teórica, sus partes, indicaciones para su aplicación e interpretación. El segundo momento fue la retroalimentación del grupo, para ello se plantearon 4 preguntas abiertas acerca de los siguientes aspectos: a) la viabilidad del instrumento, b) su utilidad, c) posibilidades de aplicación y d) sugerencias de mejora.

\subsection{Procesamiento de análisis}

Para realizar el proceso de análisis de la información se siguieron los pasos que Santos et al. (2017) retoman y sintetizan como básicos para el tratamiento de la información en un estudio desde el enfoque cualitativo. El primero de estos consiste en la obtención de la información para posteriormente, ordenarla y transcribirla. En el caso aquí expuesto, el panel de personas expertas fue por medio de la plataforma de video conferencias Zoom, el cual se grabó y escuchó en repetidas ocasiones para transcribir las participaciones.

En un segundo momento y, de acuerdo con lo expuesto por Santos et al. (2017), la información fue ordenada por medio de códigos, los cuales se establecieron, resguardando los criterios de confidencialidad. De forma que, a cada persona experta se le asignó un código para registrar sus aportes: ' $P$ ' para persona profesional y 'O' en Orientación, así como un número según el orden de ingreso a la reunión; los códigos se colocaron de PO1 a PO11.

Una vez realizada la consulta, según la finalidad del panel de expertos de llegar a concesos colectivos y profesionales acerca de un tema en concreto, se detallaron aquellas retroalimentaciones más frecuentes en las participaciones por medio de un proceso de codificación de la información, donde se utilizaron iniciales y símbolos que ayudaron a identificar patrones comunes, los cuales, según el proceso señalado por Santos et al. (2017), se compararon y agruparon para construir con ello las categorías de análisis, dos a saber: consideraciones relacionadas a aplicabilidad y utilidad y valoraciones respecto a las oportunidades de análisis que facilita el instrumento.

La primera categoría de análisis enmarca todos aquellos criterios referidos a la posibilidad de utilizar el instrumento en los distintos contextos laborales donde se desempeñan las personas profesionales en Orientación, así como su pertinencia para aplicarlo con sus 
orientadas y orientados. En la segunda categoría, se retoman todas aquellas posibilidades que ofrece el instrumento para realizar un análisis interpretativo del desarrollo de la carrera.

En el caso de los aspectos de mejora, no existe suficiente información como para generar una categoría de análisis, ya que el panel de expertos no hizo muchas modificaciones en este sentido, por lo tanto, se optó por incluirlas en las dos categorías antes citadas. Concretamente, se realizaron dos observaciones, la primera consiste en modificar la línea de la edad del Gráfico de eventos cuando trabaja con personas adolescentes, y la segunda se centra en la incorporación de la pregunta número 7 de la I Parte del Gráfico (Ver anexo 1).

Una vez realizadas las categorías se procedió a elaborar lo que Santos et al. (2017) denominan integración de la información, es decir, realizar la interpretación de los datos a partir del marco de referencia teórico del instrumento, específicamente, desde enfoque de Autoconstrucción de carrera y del enfoque de Transiciones.

\section{Hallazgos}

\subsection{Consideraciones relacionadas a la aplicabilidad y utilidad}

Como se ha mencionado, para valorar la utilidad y aplicabilidad del instrumento, este se sometió a juicio de expertos para conocer sus impresiones. Para las personas profesionales en Orientación participantes en dicho juicio, el instrumento presenta diversas ventajas que pueden obtener de su aplicación.

Entre las principales ventajas se encuentran -según las personas consultas- las siguientes:

"me parece muy bien que se puede aplicar a diversas poblaciones, adolescentes o adultas" PO8

"es sencillo de aplicar, no es complicado de comprender para la persona que lo aplique" P01

"su lenguaje es fácil de comprender y la interpretación tampoco es compleja, ni para uno ni para la persona orientada.." PO7

"es muy sencillo de aplicar y me sirve mucho para la población con la que trabajo" PO4

Al respecto McMahon y Patton (2019), señalan la importancia de ofrecer explicaciones del desarrollo de carrera capaces de integrar y sintetizar la complejidad de este proceso, y ofrecer así una mirada que describa cómo sucede este. Al respecto Sánchez (2017) indica de 
suma importancia que la persona profesional en Orientación proporcione las estrategias necesarias para una adecuada y profunda exploración de la persona orientada, en especial, en la fase diagnóstica del proceso de intervención, pues conduce mejor el resto del proceso.

En ese sentido, respecto a la importancia que señala Sánchez (2017) de los procesos exploratorios en la fase diagnóstica de la intervención, cabe señalar la necesidad de que sea la persona orientada capaz de identificar sus propios recursos y gestionarlos, comprender cómo surgen y cómo pueden serle de utilidad en medio de los cambios o exigencias que afrontar o pueden presentarse en un futuro. Al respecto, una de las personas expertas del panel señala que esto es factible con el presente instrumento de exploración:

"el instrumento es muy completo... ayuda a las personas a encontrar respuestas en ellas mismas, y no en lo que las demás y los demás puedan decir..., a diferencia de algunas herramientas cuantitativas" PO3

"me parece que al ser una exploración cualitativa donde la persona ve su desarrollo se evita que la persona deposite toda la responsabilidad por ejemplo de una decisión, en un instrumento y sus resultados... como que le devuelve la responsabilidad a ella..." PO8

Para las personas participantes, el instrumento es un insumo que contribuye mucho en su labor orientadora y expresan la necesidad de más herramientas como esta para desarrollar su trabajo, pues facilita los procesos de autoconocimiento, no solo en lo concerniente a elección de carrera u ocupación, sino en procesos de orientación para la inserción laboral, concretamente, para obtener información con el fin de delinear el perfil empleable de la persona, partiendo de sus experiencias vitales más significativas y los aprendizajes obtenidos en ellas.

"puede arrojar información de los pilares de la empleabilidad y observar sus valores ocupacionales también o la influencia de la familia...” (PO9)

"es muy útil para obtener insumos para la construcción de curriculums, en especial la parte de competencias". (PO2)

En este sentido Savickas (2015), señala que el aprendizaje es de toda la vida, igual la adaptación a los cambios, por lo que identificar dichas trayectorias facilita conocer también la condición empleable de la persona. Un instrumento de exploración como este favorece uno de 
los aspectos más importantes como lo es que, en un proceso de Orientación para el desarrollo de la carrera, la persona se comprometa con su trayectoria vital elegida por ella misma. Tal compromiso, no puede ser posible si la persona no reconoce su trayectoria, la conscientiza y la resignifica, aspecto que como se ha señalado, se facilita con la discusión que el instrumento puede generar.

Por otro lado, tal y como lo señala Sánchez (2017), el influjo de los enfoques constructivistas en Orientación está llevando a un progreso cada vez mayor de estrategias, donde la persona se vuelve protagonista de su propio proceso, por lo que se vuelve necesario generar acciones donde puedan expresar cómo se visualizan a sí mismas y comprendan su propio desarrollo, es decir, se debe buscar la manera de construir, desde la disciplina, herramientas que faciliten dicho objetivo. En este sentido una de las personas expertas señaló lo siguiente:

"este instrumento es que surge de la perspectiva orientadora y que al ser nacional no requiere de adaptaciones para su uso, casi siempre tenemos que estar adaptando instrumentos de otros lados o de otras disciplinas". PO7

Ahora bien, respecto a las limitaciones en su aplicabilidad, el grupo de expertos señaló dos aspectos en concreto. El primero de ellos relacionado con la edad de las personas orientadas y, el segundo, con la presencia de la persona Orientadora. Respecto a lo primero, señalan que por el tipo de información que se busca obtener y la necesidad de identificar varios eventos significativos y desprender aprendizajes de ellos, es pertinente tratar con personas adolescentes o adultas, incluso, si es adolescente se estima necesario adaptar el eje $\mathrm{x}$ del gráfico de los periodos de edad y no usarlo con rangos -de edad- sino con años consecutivos (de 1 año hasta 18 años), tal y como lo señalan en el siguiente comentario:

"se recomienda acortar el rango de edad del eje horizontal, de manera que no se presente en periodos de 5 años, sino del 1 al 18, siendo este uno de los aportes que pueden seguir las personas orientadoras que lo apliquen con esta población en específico" P011.

En lo que se refiere a la segunda limitación, se indica la necesidad de que la persona orientadora se encuentre presente cuando se aplica el instrumento, para que guíe a la persona orientada en la reflexión de los eventos y en las respuestas de las preguntas abiertas, tal y como lo señalan en el siguiente aporte: 
"no veo que sea un instrumento como para dejar de tarea, porque durante las reflexiones de los eventos pueden surgir situaciones y es mejor que una esté ahí por si acaso..." P010

En este sentido, y tomando como recomendación lo aportado en el panel de personas expertas, se sugiere que el instrumento se aplique bajo la supervisión de una persona profesional en Orientación.

De esta forma, como se observa, el juicio de personas expertas es favorable para el instrumento. En relación con criterios como la utilidad y viabilidad de este, lo describen como indicado y atinente para las diversas poblaciones con las que trabajan, dato muy significativo, pues se trata de un grupo heterogéneo de personas expertas, quienes además se desempeñan en diferentes ámbitos del quehacer de la Orientación.

También el grupo experto señala una serie de posibles oportunidades de análisis que puede arrojar la información del instrumento, las cuales se indican a continuación.

\subsection{Valoraciones respecto a las oportunidades de análisis que facilita el instrumento}

Una de las principales oportunidades observadas -por las personas expertas- según el tipo de información que se puede obtener del instrumento desde su carácter cualitativo, trata de la posibilidad de profundizar en la construcción de la identidad de la persona orientada, a partir de sus propias experiencias, lo cual, de acuerdo con Savickas (2015), es fundamental para ahondar en el razonamiento de la autobiografía y facilitar con ello una reconstrucción de la narrativa de la identidad. Al respecto se pueden resaltar las siguientes frases:

"es una potente herramienta para externar y resignificar las experiencias y estructura de pensamiento y ver en esas experiencias posibilidades de crecimiento". (PO10)

"Abre espacios de reflexión sobre mi vida, porque no solo emergen los eventos, sino que se pueden retomar las emociones y demás..." (PO4)

Lo anterior reafirma la intencionalidad del instrumento, en el sentido de que ayuda a operacionalizar el proceso narrativo de la construcción de carrera, enfatizado por el enfoque de Life Design de Savickas (2015), así como la comprensión de las transiciones inherentes a ese proceso sugerido por el enfoque de transiciones de Anderson et al. (2012). 
En esta misma línea, referente a la construcción de carrera por medio de la narrativa, Kapanadze (2018) menciona a este como un proceso discursivo, el cual se encuentra determinado por las diversas experiencias que viven las personas a lo largo de su vida, donde por tal razón es necesario que reflexione en su historia, para encontrar en ella lo necesario para responder a las exigencias que le presenta el medio.

Lo anterior es confirmado por Freundenthal (2017), quien señala que las personas construyen sus proyectos personales con la narración de los eventos que movilizan sus propósitos y los significados que otorgan a estos, razón por la cual se considera a la narrativa sumamente pertinente en la exploración del desarrollo de la carrera más allá de las técnicas psicométricas.

Por tal motivo, es oportuno generar estrategias como el "Gráfico de eventos significativos" para facilitar a la persona orientada la narración de sus vivencias y, al mismo tiempo, favorecer la interpretación de la persona profesional en Orientación. En otras palabras, existe una importancia en detenerse en las transiciones de la vida y en el cómo las podría resignificar para integrarlas al proyecto vital o laboral. En esta línea, una de las personas expertas del panel aporta la siguiente observación:

"el instrumento permite que las personas vean lo que han pasado, o sea sus eventos, $y$ los vean de otra manera, y le den otro sentido..." (PO3)

Es decir, la narración de las historias de vida, a partir de aquellos eventos más significativos en el desarrollo de carrera, tal y como lo pretende el presente instrumento, cumple con uno de los aspectos más importantes de los enfoques que lo sustentan, el cual trata, según Chia de la Rosa (2018), en provocar nuevas interpretaciones a algunos temas de vida, reflexionar acerca de los eventos vividos y los resultados de estos, para con ello comprender mejor las propias características personales, los recursos con los que se cuentan, así como la propia identidad personal y profesional.

Al respecto, las personas consultadas para la validación del instrumento concuerdan en la efectividad y en lo práctico para obtener información valiosa y así generar un perfil de la persona orientada en procesos de inserción laboral; en especial, durante la primera parte de la intervención, donde la finalidad se centra en comprender los intereses, destrezas y expectativas de las personas, según al mercado laboral. En ese sentido señalan: 
"es muy útil para obtener información sobre los pilares de empleabilidad, por ejemplo, es posible ver influencia de la familia y ver como concilian vida personal y profesional..." (PO6)

"nos permite obtener insumos que nos permiten conocer habilidad para el curriculum, esto es importante para la primera parte del proceso de asesoramiento para el empleo." (PO2)

Asimismo, en esta misma línea y según lo señalado por las personas expertas, una de las ventajas ofrecidas por el "Gráfico de eventos significativos", consta de facilitar, a la persona orientada, poder extraer sus destrezas de situaciones concretas de su vida y no a partir de una lista confeccionada para un curriculum, lo cual, en ocasiones se dificulta, tal y como lo señala la siguiente frase:

"en el sistema penitenciario a las personas les cuesta ver sus habilidades, ahora podrían ver cómo desde sus experiencias, aunque sean difíciles, se pueden extraer destrezas...(PO6)

En lo referente a esta oportunidad de análisis -destacada por el panel de personas experta- acerca de la información que se podría obtener para favorecer procesos de Orientación para la inserción laboral, Kapanadze (2018) afirma que, al ser la empleabilidad un constructo psicosocial, esta dependerá de hasta qué punto la persona pueda examinar su historia de vida y sus diversas transiciones, con el fin de confrontar sus aprendizajes con las exigencias del mercado laboral; lo cual concuerda con la señalado por las personas orientadoras del panel, con respecto a la aplicación que le podrían dar el instrumento.

Aunado a lo anterior, Falcón y Arraiz (2017), señalan que para la elaboración de los proyectos laborales se hace indispensable que las personas realicen una introspección donde puedan recopilar evidencias de interés significativas para ellas, así como propiciar la reflexión acerca de los aprendizajes que se pueden obtener de las experiencias vividas a lo largo del desarrollo de la carrera, todos ellos aspectos retomados con el "Gráfico de eventos significativos". 
Otra posibilidad de análisis generada con la información del instrumento consiste en la identificación de ideas, mandatos o patrones que se presenten en la exploración de los eventos y las circunstancias donde ocurrieron. Esto es respaldado por las siguientes frases:

"ahondar en estos eventos permite identificar no solo eventos, también circunstancias que bloquean a la persona, sus ideas irracionales, que no le permiten tomar decisiones..." (PO5)

“...se pueden ver ideas irracionales que no les ayuden a ver posibilidades.” (PO6)

Retomando los aportes de Falcón y Arraiz (2017) y de Chia de la Rosa (2018), concerniente con este aspecto descrito por las personas expertas, estos mandatos o ideas se relacionan con las denominadas narrativas emergentes, es decir, con aspectos transversales pertenecientes a los eventos descritos, los cuales, pueden facilitar u obstaculizar el optimismo con que se podría afrontar el proceso de transición.

Además, el aporte de Freundenthal (2017), es claro en lo relativo a estas ideas irracionales, prejuicios, temores y sentimientos de desconfianza, los cuales, desde su perspectiva, deben deconstruirse para generar nuevas alternativas y significados más motivantes y con ello nuevos caminos y oportunidades.

Por último, el instrumento podría facilitar el abordaje de aspectos como la incertidumbre frente al cambio, así como la construcción del proyecto de vida porque esto permitiría a la persona orientada visualizar eventos inesperados de onda o no eventos que hayan marcado significativamente su desarrollo y el aporte de importantes experiencias y aprendizajes. De manera que la incertidumbre afrontada en esos momentos hoy podría capitalizarse en su favor (una resignificación de las circunstancias), para adquirir un sentido en la construcción de su carrera. Según, reafirma Freundenthal (2017), considerar la incertidumbre representa para muchas personas la apertura a nuevas oportunidades, mientras que otras se atemorizan, abruman e inmovilizan frente a esta. Se podría decir que la diferencia se encuentra en la posibilidad que han tenido unas y otras para valorar sus historias de vida, los aprendizajes desprendidos de aquellos eventos no esperados, rodeados de incertidumbre y los resignificados que se puedan construir alrededor de estos.

A partir de estos criterios profesionales de personas orientadoras que laboran en diversos contextos de intervención, es posible afirmar que el "Gráfico de eventos del desarrollo" de la Carrera, gracias a su carácter cualitativo, responde a lo que las nuevas 
perspectivas en Orientación plantean, acerca de la necesidad de ajustar la intervención de manera no lineal o predictiva, debido a que el mundo en sí mismo es incierto, no lineal y cambiante.

En síntesis, el "Gráfico de eventos significativos en el desarrollo de la carrera", permite como instrumento no solo operacionalizar el proceso narrativo del desarrollo de carrera, sino también facilitar el asesoramiento de la persona en transición, donde se comprende que, como lo mencionan Anderson et al. (2012), es necesario indagar en aspectos como los cambios, las ganancias, las pérdidas, más allá del mero ajuste, y pasar a comprender las transiciones como eventos transformadores que devuelven el sentido de vida a las personas.

\section{Conclusiones}

Una vez descrito y analizado el instrumento cualitativo de exploración para el Gráfico de eventos del desarrollo de la carrera", se concluye:

El instrumento de exploración como tal, permite durante los procesos de intervención orientadora, profundizar en la comprensión de la construcción de la identidad personal y profesional, a partir de los eventos más significativos de la persona, gracias a la resignificación de estos, con la identificación de los principales aprendizajes y apoyos disponibles.

El gráfico facilita la operacionalización del análisis de transiciones propuesto por el enfoque de transiciones, así como de la narrativa de las historias de vida que plantea el enfoque de autoconstrucción de la carrera.

Se sugiere que este instrumento se utilice con el objetivo de delimitar un perfil de la persona orientada para guiar la dirección del proceso de intervención con sus características e historia de vida.

Este instrumento exploratorio reduce el tiempo de indagación de características personales y contextuales de la persona orientada, pues puede realizarse en un tiempo estimado de una o dos sesiones de trabajo.

La información generada por el instrumento es considerada como valiosa por las personas expertas, tanto para ser utilizada en procesos, cuya finalidad sea la elección de una profesión u ocupación, así como para procesos de asesoramiento para la inserción laboral.

El "Gráfico de eventos significativos del desarrollo de la carrera" puede ser utilizado en poblaciones adolescentes o adultas, en educación formal y no formal, en el sistema penitenciario, así como en otros ámbitos de intervención. 
Su aplicación puede realizarse de manera individual o colectiva. En el caso de las sesiones grupales, se recomienda que la persona profesional en Orientación facilite un ambiente adecuado para la retroalimentación de las personas orientadas y el respeto por el desarrollo de carrera de quienes participan.

Presenta la ventaja de ser un instrumento de exploración generado desde la perspectiva de Orientación y en el contexto nacional, el cual responde a las recomendaciones de los enfoques más recientes, respecto al desarrollo de la carrera, donde se señala la necesidad de una exploración enfocada cada vez más en los significados de los eventos personales y no tanto en las intervenciones lineales y de carácter predictivo, como suele ocurrir en el uso de las herramientas cuantitativas.

Dentro de las limitaciones del "Gráfico de eventos de significativos del desarrollo de la carrera", se pueden mencionar:

Debido a la intencionalidad y a que la fundamentación teórica utilizada en la construcción surge, principalmente, desde la disciplina de la Orientación, su aplicación debe ser realizada por profesionales en esta área, esto con el fin de garantizar una asesoría consistente con el área disciplinar desde donde se plantea el instrumento.

El diálogo que se propicie con la información obtenida con el instrumento, al ser de carácter cualitativo, dependerá de la destreza de la persona orientadora, los objetivos que persiga con su empleo y los emergentes surgidos en el proceso.

El gráfico requiere ser ajustado en el eje $\mathrm{x}$, relativo con la edad, cuando se aplique en personas adolescentes, pues está elaborado en rangos de 5 años, los cuales, pueden ser útiles con adultos, pero no con aquellas más jóvenes, por lo tanto, es necesario enumerar los años del 1 al 18 consecutivamente, para ubicar los eventos significativos.

Dicho lo anterior, no se recomienda el uso de este instrumento con población en edad infantil, pues se considera que no aportaría información suficiente acerca de eventos significativos para el análisis del desarrollo de carrera.

Finalmente, para la aplicación del instrumento, se sugiere que la persona profesional en Orientación esté presente mientras la persona orientada lo elabora, con el objetivo de acompañarla en su reflexión personal y contener posibles situaciones emergentes que requieren de una intervención pronta y oportuna. Por esto, no se recomienda dejar el instrumento como una asignación para realizar en casa y sin supervisión. 


\section{Referencias}

Anderson, Mary., Goodman, Jean., y Schlossberg, Nancy (2012) Counseling Adults in transitions. Linking Schlossberg theory with practice in adverse world ( $4^{\text {th }}$ ed.). Spencer Publishing Company.

Bisquerra, Rafael. (2016). Orígenes y desarrollo de la orientación pedagógica. Madrid, España: Narcea Ediciones. Recuperado de https://elibronet.ezproxy.sibdi.ucr.ac.cr/es/ereader/sibdi/45930?page $=40$

Chia de la Rosa, Paula. (2018) Trayectoria, motivación y adaptabilidad de la carrera en los estudiantes de máster de educación en la Universidad de Sevilla (Tesis de Grado en Pedagogía). Universidad de Sevilla, Sevilla, España. https://idus.us.es/bitstream/handle/11441/81800/174 47556657.pdf?sequence=1\&isAll owed=y

Falcón Carolina., y Arraiz Ana. (2017). Construcción eficiente y sostenible de la carrera: el portafolio como recurso de orientación universitaria. REOP - Revista Española De

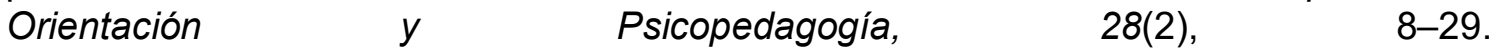
https://doi.org/10.5944/reop.vol.28.num.2.2017.20116

Freudenthal, Erika. (2017). De-construcción, re-construcción, co-construcción de la carrera profesional para un contexto no-occidental. Revista de Investigacion Psicologica, (17), 105-116. $\quad$ http://www.scielo.org.bo/scielo.php?script=sci arttext\&pid=S2223$\underline{30322017000100007}$

Fuster, Doris. (2019). Investigación cualitativa: Método fenomenológico hermenéutico. Revista $\begin{array}{llll}\text { Propósitos } \quad y \quad \text { Representaciones 201-229. Doi } & \end{array}$ http://dx.doi.org/10.20511/pyr2019.v7n1.267

Guichard, Jean. (2002). Problemáticas y finalidades de la orientación profesional. Revista Europea en Formación Profesional, (26), 5-2.

Guichard, Jean. (2007). Life-long self-construction: A core issue of vocational psychology in a globalized world. In J. Guichard (Convener), Vocational guidance and diversity across the world. Invited symposium presented at IAEVG international conference, Padova, Italy, September 4-6.

Kapanadze, Maria. (2018) Moral identity and carrer construction: implications for theory intervention and research. Example of people in recovery from substance use disorders (Doctoral Thesis). Universitat de Girona, Girona, España. http://hdl.handle.net/10803/663725

Kvale, Steinar. (2011). Doing Interview. Ediciones Morata.

Lewthwaite, Sara., y Nind, Melanie. (2016). Teaching Research Methods in the Social Sciences: Expert Perspectives on Pedagogy and Practice. British Journal of Educational Studies, 64(4), 413-430. 
López, Inmaculada., Blanco, Ascension., Pagán, Rosa., Gazapo, Bienvenido., Arana, José., Pizarro Ester., y Martínez, Beatriz. (2014). Estudio cualitativo sobre tutoría universitaria a través del método del panel de expertos. Higher Learning Research Communications, 4(1), 73-90. https://bit.ly/3yvCllk

McMahon, Mary. (2014). New Trends in Theory Development in Career Psychology. En Gideon Arulmani, Anuradha Bakshi, Frederick Leong and A. Watts (Eds.), Handbook of Career Development. International Perspectives (pp. 13-28). Springer.

McMahon, Mary, y Patton, Wendy. (2019). The Systems Theory Framework: A Systems Map for Career Theory, Research and Practice. In James A. Athanasou, y Harsha Perera (Eds.), Internatinal Handbook Carrer Guidance (pp. 97-114). Swizterland: Springer Science.

Ribeiro, Marcelo. (2013). Reflexiones epistemológicas para la orientación profesional en América Latina: una propuesta desde el Construccionismo Social. Revista Mexicana de Orientación, 24(10), 2-10.

Robles, Pilar., y Rojas, Manuela. (2015). La validación por juicio de expertos: dos investigaciones cualitativas en Lingüística aplicada. Revista Nebrija de Lingüistica, (18). https://www.nebrija.com/revista-linguistica/la-validacion-por-juicio-de-expertos-dosinvestigaciones-cualitativas-en-linguistica-aplicada.html

Sánchez, Fabio. (2019). Fundamentos epistémicos de la investigación cualitativa y cuantitativa: consensos y disensos. Revista Digital de Investigación en Docencia Universitaria, 13(1), 102-122. doi https://doi.org/10.19083/ridu.2019.644

Sánchez, María Fe. (2017). Orientación para el desarrollo profesional. San José, Costa Rica: Editorial de la Universidad Estatal a Distancia. https://elibronet.ezproxy.sibdi.ucr.ac.cr/es/ereader/sibdi/48917?page=64

Santos Ofelia., Hidalgo Cristian., y Arreaga, Carlos. (2017). La etapa final del análisis y la redacción del informe de investigación cualitativo. En Carlos Escudero y Liliana Cortéz, Técnicas y Métodos Cualitativos en la Investigación Científica (pp. 90-104). Ecuador: Editorial UTCHMAN.

Savickas, Mark. (2015). Life Desing Counseling Manual. Mark Savickas. http://www.vocopher.com/LifeDesign/LifeDesign.pdf

Savickas, Mark., Nota, Laura., Rossier, Jerome., Dauwalder, Jean., Duarte, María., Guichard, Jean., Soresi, Salvatore., Esbroeck, Raoul. and Van Vianen, Annelies. (2009). Construir su vida (Life Designing). Un paradigma para la Orientación. Journal of Vocational Behavior, 75(3), 239-250 Trad. Diana Aisenson y Gabriela Aisenson. https://researchportal.vub.be/en/publications/life-designing-a-paradigm-forcareer-construction-in-the-21st-cen

Super, Donald. (1969). Vocational Development Theory: Persons, Positions and Processes. The Counseling Psychologist, 1(1), 2-9. 


\begin{abstract}
Anexo 1
Instrumento cualitativo de exploración: Gráfico de Eventos en el Desarrollo de Carrera.

Descripción General: El presente instrumento tiene como finalidad explorar los principales eventos que han influido en la persona durante su desarrollo de carrera, de manera que se pueda establecer, a partir de estos y el significado que la persona les asigne, un perfil de la persona orientada. Con dicho perfil se pretende tener información personal clave para establecer los lineamientos del proceso de intervención, favorecer el autoconocimiento y la toma de decisiones.
\end{abstract}

\title{
Indicaciones:
}

\section{PARTE}

1. Seleccione 5 eventos significativos en su vida: estos pueden haber sido satisfactorios o no. Puede seleccionar eventos de índole académico (graduaciones, éxito en un examen o reprobar un año), profesional (conseguir un empleo, perder un empleo, no obtener un ascenso, obtener una distinción, entre otros) o eventos en la vida personal (la separación de sus padres, un paseo, una pérdida, conocer una persona, aprender un instrumento u otros).

2. Ubique estos eventos en la línea del tiempo: ¿a qué edad sucedió cada evento?

3. Clasifique los eventos de acuerdo con los siguientes criterios:

Un evento Esperado: es aquel que usted anhelaba y por el que se esforzó por que sucediera y aconteció.

De onda: es un evento que sucedió a un tercero, pero tuvo un impacto significativo en su vida (por ejemplo, el divorcio de sus padres o trasladarse de lugar porque su madre o padre cambiaron de empleo)

Inesperado: es un evento que impactó significativamente su vida, pero no fue algo planificado.

No evento: es aquel que usted anhelaba y por el que se esforzó por que sucediera, pero no aconteció (por ejemplo, no quedar embarazada, no obtener un ascenso).

4. Ubique los eventos en el gráfico: señale con un punto donde se cruza el tipo de evento, con la edad en la que este sucedió. 


\section{Gráfico de eventos significativos en el desarrollo de la carrera:}

\section{Tipo de evento}

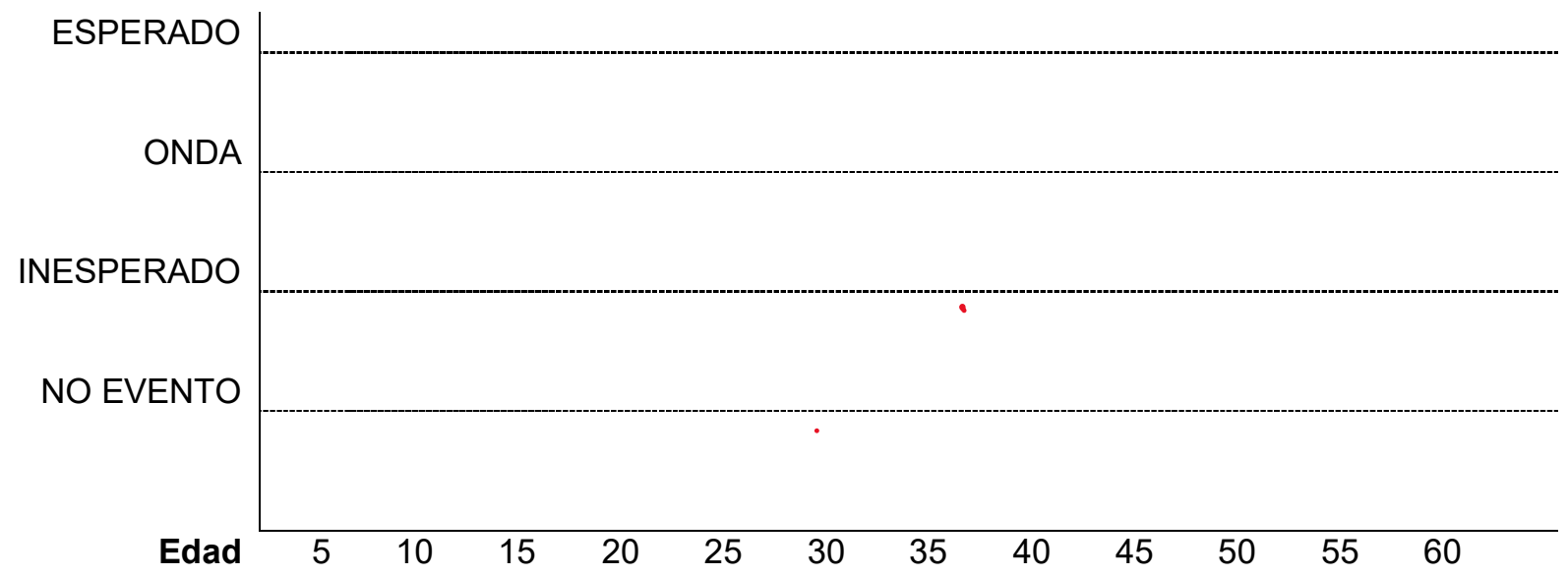

Fuente: Elaboración propia

5. Luego, con una línea, una los puntos.

6. Reflexione acerca de los tipos de eventos que han sido más significativos en su vida, la edad en que sucedieron y la influencia que han tenido en la conformación de su identidad.

7. ¿Considera algún evento de los destacados más significativo que los otros? ¿Por qué razón?

\section{PARTE}

Reflexione y responda las siguientes preguntas, de acuerdo con su experiencia en cada uno de los eventos mencionados, anote cada respuesta en el espacio que corresponda en la matriz que se le presenta:

1. ¿Qué aprendizaje(s) puedo rescatar de este evento para mi vida?

2. ¿Con qué apoyos conté para afrontar esos eventos? ¿Familia, amigos, instituciones, docentes? ¿Permanecen estos apoyos?

3. ¿Qué aportaron estos apoyos a mi vida?

4. A partir de estas experiencias y aprendizajes ¿Qué destrezas o habilidades desarrollé a partir de este evento? 


\begin{tabular}{|l|l|c|c|}
\hline Eventos & $\begin{array}{c}\text { Aprendizajes ¿puedo } \\
\text { rescatar de este } \\
\text { evento? }\end{array}$ & $\begin{array}{c}\text { Apoyos } \\
\text { ¿qué aportes me } \\
\text { brindaron estos apoyos? }\end{array}$ & $\begin{array}{c}\text { Destrezas o habilidades } \\
\text { ¿Qué destrezas o } \\
\text { habilidades desarrollé a } \\
\text { partir de este evento? }\end{array}$ \\
\hline & & & \\
\hline & & & \\
\hline & & & \\
\hline & & & \\
\hline
\end{tabular}

Fuente: Elaboración propia

Retome los aspectos señalados en la columna de la Matriz, llamada Destrezas y complete la siguiente frase:

Gracias a estas experiencias vitales, soy una persona:

Por último:

¿Qué opinión le merece esta información personal?

Retome la información del instrumento y discútala con su profesional en Orientación. 
Revista indizada en

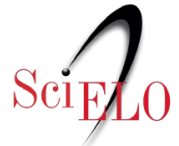

redalyc.sy latindex

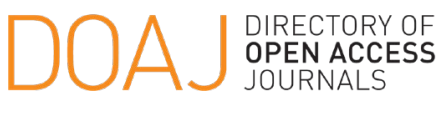

Distribuida en las bases de datos:
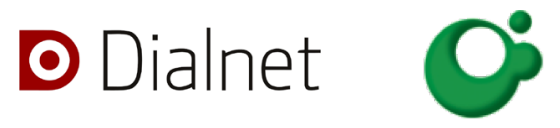
SHERPA/RøMEO

REDIB

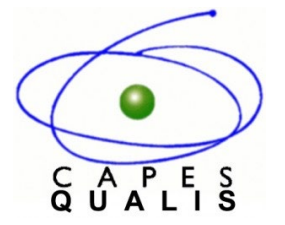

MIAR 